\title{
ANĀLISIS DE VIGAS POR ELEMENTOS FINITOS
}

\author{
Cristian Loli Prudencio ${ }^{1}$, Edwin Chávez Ramírez ${ }^{2}$, Victoriano Yauri Luque ${ }^{3}$, \\ Andrés Guardia Cayo ${ }^{4}$, Amelia Villanueva Yaya ${ }^{5}$, Juan Luna Valdez ${ }^{6}$
}

(Recibido: 20/08/2014 - Aceptado: 30/09/2014)

Resumen: Este trabajo presenta el análisis de deformación de las vigas a través del modelo definido en el intervalo $[a, b]$

$$
\begin{gathered}
-\frac{d}{d x}\left(p(x) \frac{d y}{d x}\right)+q(x) y=f(x) \\
r_{0} u^{\prime}(a)+s_{0} u(a)=c_{0} \\
r_{1} u^{\prime}(b)+s_{1} u(b)=c_{1}
\end{gathered}
$$

Este modelo está asociado a las vigas simplemente apoyadas, empotradas y en voladizo, sujetas a cargas distribuidas diversas e inclusive con sección transversal variable.

La solución se hace pasando el modelo a la forma débil y luego a la forma discreta. La forma discreta se resuelve en un espacio finito dimensional, este espacio será el espacio de los elementos finitos lineales para este trabajo, y la solución nos arroja la deflexión de la viga a lo largo de toda su extensión (luz).

Palabras clave: Dirichlet, Newmann, Mixto, Solución Débil, Problema Discreto, Forma Variacional, Elementos Finitos Lineales, Simulación.

\section{ANALYSIS OF BEAMS BY FINITE ELEMENT}

Abstract: This paper presents the analysis of deformation beams through the model defined on the interval $[a, b]$

$$
\begin{gathered}
-\frac{d}{d x}\left(p(x) \frac{d y}{d x}\right)+q(x) y=f(x) \\
r_{0} u^{\prime}(a)+s_{0} u(a)=c_{0} \\
r_{1} u^{\prime}(b)+s_{1} u(b)=c_{1}
\end{gathered}
$$

This model is associated with simply supported beams, Recessed and cantilever subject to various loads and distributed even with variable cross section.

The solution is passing the model to the weak form and then the discrete form. the discreetly out on a finite dimensional space, this space is the linear space for this finite element work, and the solution yields us the deflection of the beam along its entire length (light).

Key words: Dirichlet, Newmann, Mixed, Weak Solutions, Discrete Problem, Variational Shape, Linear Finite Element, Simulation.

\footnotetext{
${ }^{1}$ UNMSM, Facultad de Ciencias Matemáticas, e-mail: clolip@unmsm.edu.pe

${ }^{2}$ UNMSM, Facultad de Ciencias Matemáticas, e-mail: edwin.chavez@unmsm.edu.pe

${ }^{3}$ UNMSM, Facultad de Ciencias Matemáticas, e-mail: vyauril@unmsm.edu.pe

${ }^{4}$ UNMSM, Facultad de Ciencias Matemáticas, e-mail: agcbayo@yahoo.es

${ }^{5}$ UNMSM, Facultad de Ciencias Matemáticas, e-mail: avillanuevay@unmsm.edu.pe

${ }^{6}$ UNMSM, Facultad de Ciencias Matemáticas, e-mail: jlunav@unmsm.edu.pe
} 


\section{Introducción}

El método de los elementos finitos (MEF) ha adquirido una gran importancia en la solución de problemas de ingeniería, ya que permite resolver casos que hasta hace poco tiempo eran prácticamente imposibles de resolver por métodos matemáticos tradicionales.

Esta circunstancia obligaba a realizar prototipos, ensayarlos e ir realizando mejoras de forma iterativa, lo que traía consigo un elevado coste tanto económico como en tiempo de desarrollo.

Consideremos el siguiente problema, dado $f \in C([a, b])$, hallar una función $u(x)$ definida en el intervalo $[a, b]$ tal que

$$
\mid \begin{gathered}
-u^{\prime \prime}+u=f \\
u(a)=u(b)=0
\end{gathered}
$$

Una solución clásica o solución fuerte del problema es una función de clase $C^{2}[a, b]$ que verifica (1) en el sentido usual. Claro esta, (1) se puede resolver explícitamente con un calculo muy sencillo, pero ignoraremos este aspecto con el fin de ilustrar el método de elementos finitos a partir de este ejemplo elemental.

Se multiplica (1) por $\phi \in C_{0}^{1}([a, b])$ y se integra por partes, de donde resulta

$$
\int_{a}^{b}\left(u^{\prime} \phi^{\prime}+u \phi\right) d x=\int_{a}^{b} f \phi d x, \forall \phi \in C_{0}^{1}([a, b])
$$

Observése que (2) tiene sentido si $u \in C_{0}^{1}([a, b])$ (que es menos requisito que (1), que supone a $u$ derivable dos veces), de hecho incluso seria suficiente tener $u, u^{\prime} \in L^{1}([a, b])$ donde $u^{\prime}$ es la derivada débil (distribucional).

La solución discreta se toma a través del espacio finito dimensional de funciones

$$
V_{h}=\operatorname{Gen}\left\{\phi_{i} \in C([a, b]) / \phi_{i}(a)=\phi_{i}(b)=0, i=1,2, . ., N\right\}
$$

de tal forma que la solución aproximada $u_{h} \in V_{h}$ cumple la ecuación

$$
\int_{a}^{b}\left(u_{h}^{\prime} \phi_{i}^{\prime}+u_{h} \phi_{i}\right) d x=\int_{a}^{b} f \phi_{i} d x, \quad \forall \phi_{i} \in V_{h}
$$

Consideremos otro problema. Dado $f \in L^{2}([0,1])$, hallar una función $u(x)$ definida en el intervalo $[0,1]$ tal que

$$
\mid \begin{gathered}
-u^{\prime \prime}+u=f \\
u(0)=0, u^{\prime}(1)=3
\end{gathered}
$$

Sea

$$
V=H_{<0}(0,1)=\left\{v \in H^{1}(0,1) / v(0)=0\right\}
$$

La formulación débil de este problema es, para $u$ en $V$

$$
\int_{0}^{1}\left(u^{\prime} \phi^{\prime}+u \phi\right) d x=\int_{0}^{1} f \phi d x+3 \phi(1), \quad \forall \phi \in V
$$

Sea

$$
V_{h}=\operatorname{Gen}\left\{\phi_{i} \in C([0,1]) / \phi_{i}(0)=0, i=1,2, . ., N\right\}
$$

de tal forma que la solución aproximada (solución discreta) $u_{h} \in V_{h}$ cumple la ecuación

$$
\int_{0}^{1}\left(u_{h}^{\prime} \phi_{i}^{\prime}+u_{h} \phi_{i}\right) d x=\int_{0}^{1} f \phi_{i} d x+3 \phi_{i}(1), \quad \forall \phi_{i} \in V_{h}
$$

Como vemos a través de los problemas (1) y (4) se puede llegar a plantear la formulación discreta (3) y (6) que con el espacio de elementos finitos se podrá resolver los problemas en forma aproximada vía una combinación lineal de los elementos de la base. Obtener esta solución aproximada es uno de los objetivos del presente trabajo. 


\section{Solución por Elementos Finitos Lineales}

Consideremos ahora un problema más general. Hallar una función $u(x)$ definida en el intervalo $[a, b]$ tal que

$$
\begin{gathered}
-\left(p(x) u^{\prime}\right)^{\prime}+q(x) u^{\prime}+r(x) u=f(x) \\
r_{0} u^{\prime}(a)+s_{0} u(a)=c_{0} \\
r_{1} u^{\prime}(b)+s_{1} u(b)=c_{1}
\end{gathered}
$$

Donde $r_{0}, r_{1}, s_{0}, s_{1}, c_{0}, c_{1}$ son constantes dadas.

Hacemos la integración por partes obteniendo así su forma variacional. Consideramos el espacio finito dimensional

$$
V_{h}=\operatorname{Gen}\left\{\phi_{1}, \phi_{2}, \ldots, \phi_{N}\right\}
$$

Las funciones de base son:

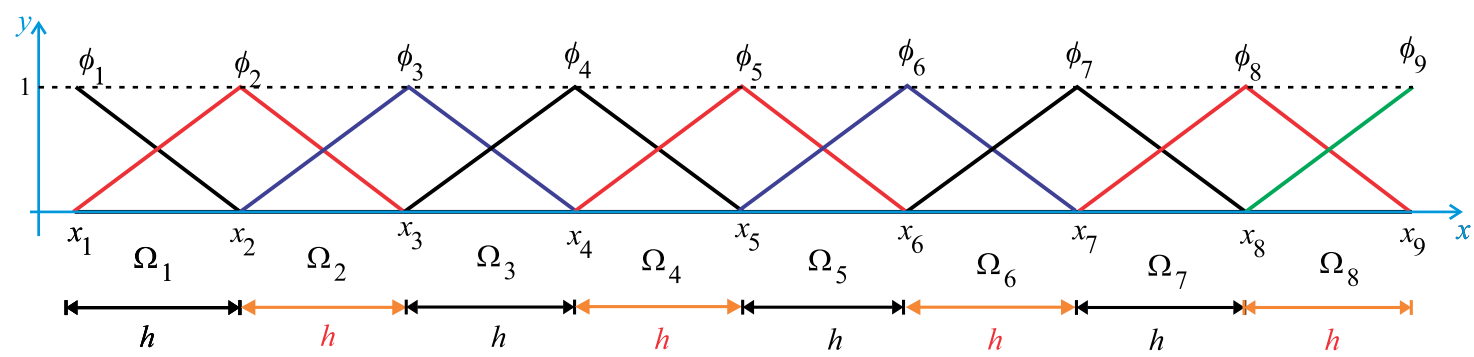

con lo cual para $u_{h} \in V_{h}$ se obtiene la formulación discreta

$$
\int_{a}^{b}\left(p u_{h}^{\prime} v^{\prime}+q u_{h}^{\prime} v+r u_{h} v\right) d x=\int_{a}^{b} f(x) v d x+\left(\left.p u_{h}^{\prime} v\right|_{a} ^{b}, \quad \forall v \in V_{h}\right.
$$

Las condiciones de frontera contienen términos con derivadas y hacemos que estas condiciones formen parte del planteamiento, esto es:

$$
\int_{a}^{b}\left(p u_{h}^{\prime} v^{\prime}+q u_{h}^{\prime} v+r u_{h} v\right) d x=\int_{a}^{b} f v d x+p(b)\left[\frac{c_{1}-s_{1} u_{h}(b)}{r_{1}}\right] v(b)-p(a)\left[\frac{c_{0}-s_{0} u_{h}(a)}{r_{0}}\right] v(a)
$$

En el caso de que las condiciones de frontera, no contengan términos con derivadas, por ejemplo sean de la forma $s_{0} u(a)=c_{0}, s_{1} u(b)=c_{1}$

Entonces se considerarán las condiciones de frontera esenciales $v(a)=v(b)=0$ con lo que la formulación se convierte en

$$
\begin{gathered}
\int_{a}^{b}\left(p u_{h}^{\prime} v^{\prime}+q u_{h}^{\prime} v+r u_{h} v\right) d x=\int_{a}^{b} f(x) v d x, \quad v \in \operatorname{Gen}\left\{\phi_{1}, \phi_{2}, \ldots, \phi_{N}\right\} \\
u_{h}(a)=\frac{c_{0}}{s_{0}}, \quad u_{h}(b)=\frac{c_{1}}{s_{1}}, \quad v(a)=v(b)=0
\end{gathered}
$$

Condiciones como estas (que no contienen derivadas) se llaman esenciales y no figuran en el modelo discreto.

Analizaremos el elemento típico $\Omega_{k}$

$\Omega_{k}$ definido como el intervalo $\left(x_{k}, x_{k+1}\right)$ para dos nodos consecutivos $x_{k}, x_{k+1}$.

$$
\int_{x_{k}}^{x_{k+1}}\left(p u_{h}^{\prime} v^{\prime}+q u_{h}^{\prime} v+r u_{h} v\right) d x=\int_{x_{k}}^{x_{k+1}} f v d x+p\left(x_{k+1}\right) u_{h}^{\prime}\left(x_{k+1}\right) v\left(x_{k+1}\right)-p\left(x_{k}\right) u_{h}^{\prime}\left(x_{k}\right) v\left(x_{k}\right)
$$


Deseamos una solución aproximada $u_{h}^{e}$ en este elemento, para esto se considera dos nodos por elementos, que significa que tendremos dos funciones de forma diferentes $\phi_{1}^{e}(x)$ y $\phi_{2}^{e}(x), \mathrm{y}$ con ello tenemos

$$
u_{h}^{e}(x)=\sum_{j=1}^{2} w_{j}^{e} \phi_{j}^{e}(x)
$$

donde $e$ indica el elemento; sustituyendo en el planteamiento tenemos :

$$
\sum_{j=1}^{2} A_{i j}^{e} \phi_{j}^{e}=f_{i}^{e}+p\left(x_{k+1}\right) u_{h}^{\prime}\left(x_{k+1}\right) \phi_{i}^{e}\left(x_{k+1}\right)-p\left(x_{k}\right) u_{h}^{\prime}\left(x_{k}\right) \phi_{i}^{e}\left(x_{k}\right)
$$

donde $\mathrm{i}=1,2$

$$
A_{i j}^{e}=\int_{x_{k}}^{x_{k+1}}\left[p \phi_{i}^{\prime} \phi_{j}^{\prime}+q \phi_{i} \phi_{j}^{\prime}+r \phi_{i} \phi_{j}\right] d x \quad y \quad f_{i}^{e}=\int_{x_{k}}^{x_{k+1}} f \phi_{i} d x
$$

Con esto se puede calcular la matriz de rigidez local $\mathrm{A}^{e}$ y el vector parcial de carga local $\mathrm{F}^{e}$.

$$
A^{e}=\left(\begin{array}{cc}
A_{11}^{e} & A_{12}^{e} \\
A_{21}^{e} & A_{22}^{e}
\end{array}\right), F^{e}=\left(\begin{array}{c}
f_{1}^{e} \\
f_{2}^{e}
\end{array}\right)
$$

Con estas consideraciones supongamos que tenemos $\mathrm{N}$ nodos ( $\mathrm{N}-1$ elementos), por lo tanto la matriz de rigidez global A será la dimensión $\mathrm{N}$ x N y el vector de carga de $\mathrm{N}$ elementos.

Veamos como se forma esta matriz.

Para el elemento $\Omega_{1}$

$$
\begin{gathered}
\text { Nodo } 1: A_{11}^{1} w_{1}+A_{12}^{1} w_{2}=f_{1}^{1}-p(a) u_{h}^{\prime}(a) \\
\text { Nodo2 }: A_{21}^{1} w_{1}+A_{22}^{1} w_{2}=f_{2}^{1}+p\left(x_{2}\right) u_{h}^{\prime}\left(x_{2}\right)
\end{gathered}
$$

\section{Para el elemento $\Omega_{2}$}

$$
\begin{aligned}
& \text { Nodo2 }: A_{11}^{2} w_{2}+A_{12}^{2} w_{3}=f_{1}^{2}-p\left(x_{2}\right) u_{h}^{\prime}\left(x_{2}\right) \\
& \text { Nodo3: } A_{21}^{1} w_{2}+A_{22}^{1} w_{3}=f_{2}^{2}+p\left(x_{3}\right) u_{h}^{\prime}\left(x_{3}\right)
\end{aligned}
$$

Ensamblando estas ecuaciones obtenemos:

$$
\begin{gathered}
A_{11}^{1} w_{1}+A_{12}^{1} w_{2}=f_{1}^{1}-p(a) u_{h}^{\prime}(a) \\
A_{21}^{2} w_{1}+\left(A_{11}^{2} w_{2}+A_{22}^{1}\right) w_{2}+A_{22}^{2} w_{3}=f_{2}^{1}+f_{1}^{2} \\
A_{21}^{2} w_{2}+A_{22}^{2} w_{3}=f_{2}^{2}+p\left(x_{3}\right) u_{h}^{\prime}\left(x_{3}\right)
\end{gathered}
$$

Así finalmente consideramos los últimos nodos , N-2, N-1 y N.

$$
\begin{array}{ll}
\text { Nodo } & N-2: A_{11}^{N-2} w_{N-2}+A_{12}^{N-2} w_{N-1}=f_{1}^{N-2}-p\left(x_{N-2}\right) u_{h}^{\prime}\left(x_{N-2}\right) \\
\text { Nodo } & N-1: A_{21}^{N-2} w_{N-2}+A_{22}^{N-2} w_{N-1}=f_{2}^{N-2}+p\left(x_{N-1}\right) u_{h}^{\prime}\left(x_{N-1}\right)
\end{array}
$$

Para el elemento $\Omega_{N-1}$

$$
\begin{gathered}
\text { Nodo } N-1: A_{11}^{N-1} w_{N-1}+A_{12}^{N-1} w_{N}=f_{1}^{N-1}+p\left(x_{N-1}\right) u_{h}^{\prime}\left(x_{N-1}\right) \\
\quad \text { Nodo } \quad N: A_{21}^{N-1} w_{N-1}+A_{22}^{N-1} w_{N}=f_{2}^{N-1}+p(b) u_{h}^{\prime}(b)
\end{gathered}
$$

Con esto la matriz global o de Ensamblaje será de la forma:

$$
A=\left(\begin{array}{lllll}
A_{11}^{1} & A_{12}^{1} & 0 & \ldots & 0 \\
A_{21}^{1} & A_{11}^{1}+A_{22}^{2} & A_{12}^{2} & 0 \ldots & 0 \\
0 & A_{21}^{2} & \ddots & \ddots & \vdots \\
\vdots & 0 & \ddots & A_{22}^{N-1}+A_{11}^{N-1} & A_{12}^{N-1} \\
0 & 0 \ldots & 0 & A_{21}^{N-1} & A_{22}^{N-1}
\end{array}\right)
$$


Y el correspondiente vector de carga será:

$$
F=\left(\begin{array}{l}
f_{1}^{1}-p(a) u_{h}^{\prime}(a) \\
f_{2}^{1}+f_{1}^{2} \\
f_{2}^{2}+f_{1}^{3} \\
f_{2}^{3}+f_{1}^{4} \\
\vdots \\
f_{2}^{N-2}+f_{1}^{N-1} \\
f_{2}^{N-1}+p(b) u_{h}^{\prime}(b)
\end{array}\right)
$$

Como podemos observar la obtención de $A$ y $F$ ha sido independiente de las condiciones en la frontera $a$ y $b$.

Si consideramos las condiciones de frontera se modifican: los elementos de las posiciones $(1,1)$ y $(\mathrm{N}, \mathrm{N})$ de la matriz $A$, y, el primero y el último elemento de $F$.

Veamos dos casos:

\section{i) Con las condiciones generales:}

$$
\mid \begin{aligned}
& r_{0} u^{\prime}(a)+s_{0} u(a)=c_{0} \\
& r_{1} u^{\prime}(b)+s_{1} u(b)=c_{1}
\end{aligned}
$$

la solución aproximada deberá satisfacer

$$
\mid \begin{gathered}
r_{0} u_{h}^{\prime}(a)+s_{0} u_{h}(a)=c_{0} \\
r_{1} u_{h}^{\prime}(b)+s_{1} u_{h}(b)=c_{1}
\end{gathered}
$$

con esto $A$ y $F$ varían de la forma siguiente:

$$
\begin{gathered}
A_{11}^{1} \quad \text { se convierte en } A_{11}^{1}-p(a) \frac{s_{0}}{r_{0}} \\
A_{22}^{N-1} \quad \text { se convierte en } A_{22}^{N-1}+p(b) \frac{s_{1}}{r_{1}} \\
f_{1}^{1}-p(a) u_{h}^{\prime}(a) \quad \text { se convierte en } f_{1}^{1}-p(a) \frac{c_{0}}{r_{0}} \\
f_{2}^{N-1}+p(b) u_{h}^{\prime}(b) \quad \text { se convierte en } f_{2}^{N-1}+p(b) \frac{c_{1}}{r_{1}}
\end{gathered}
$$

ii) Con las condiciones esenciales (Dirichlet):

$$
\mid u(a)=\frac{c_{0}}{s_{0}} \quad, u(b)=\frac{c_{1}}{s_{1}}
$$

la solución aproximada deberá satisfacer

$$
u_{h}(a)=\frac{c_{0}}{s_{0}} \quad, u_{h}(b)=\frac{c_{1}}{s_{1}}
$$

Se tienen $w_{1}=u_{h}(a)=u(a)$ y $w_{N}=u_{h}(b)=u(b)$ con lo cual sólo hay $N-2$ incógnitas que hallar $w_{2}, w_{3}, \ldots, w_{N-1}$. Para ello removemos la primera y última ecuación del sistema y en consecuencia, los efectos de estas filas en las demás filas pasarán al término independiente, esto es se eliminan la primera y la última columna también.

Con esto la segunda y $(N-1)$-ésima ecuación serán:

2da ecuación:

$$
\begin{aligned}
A_{21}^{1} w_{1}+\left(A_{11}^{2}+A_{22}^{1}\right) w_{2}+A_{12}^{2} w_{3} & =f_{2}^{1}+f_{1}^{2} \quad \text { se convierte en } \\
\left(A_{11}^{2}+A_{22}^{1}\right) w_{2}+A_{12}^{2} w_{3} & =f_{2}^{1}+f_{1}^{2}-A_{21}^{1} \frac{c_{0}}{s_{0}}
\end{aligned}
$$

$N-1$ ecuación:

$$
\begin{gathered}
A_{21}^{N-2} w_{N-2}+\left(A_{11}^{N-1}+A_{22}^{N-2}\right) w_{N-1}+A_{12}^{N-1} w_{N}=f_{2}^{N-1}+f_{1}^{N} \quad \text { se convierte en } \\
A_{21}^{N-2} w_{N-2}+\left(A_{11}^{N-1}+A_{22}^{N-2}\right) w_{N-1}=f_{2}^{N-1}+f_{1}^{N}-A_{21}^{N-1} \frac{c_{1}}{s_{1}}
\end{gathered}
$$




\section{Simulación}

Se tiene el modelo

$$
\begin{gathered}
-y^{\prime \prime}+y=x \operatorname{sen}(x) \\
y(0)=2 \\
y^{\prime}(1)=3
\end{gathered}
$$

Tomando una partición de 8 subintervalos del mismo tamaño en [0,1], por elementos finitos lineales, calcularemos la matriz de ensamblaje y el vector de carga, averiguaremos el valor de la deflexión (solución) en cada nodo, y la función solución aproximada.

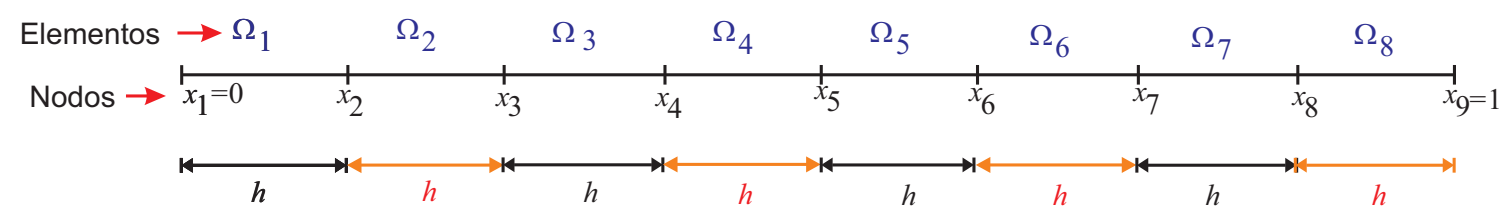

De la ecuación identificamos las siguientes funciones:

$$
p(x)=1 ; q(x)=1 \quad ; \quad f(x)=x \operatorname{sen}(x)
$$

Partimos el intervalo $[0,1]$ en 8 subintervalos iguales cuyos extremos son los nodos $\mathrm{x}_{i}=(i-1) h$, para $i=1,2, \ldots, 9$ donde $\mathrm{h}=\frac{1-0}{8} \Rightarrow h=\frac{1}{8}=0,125$. Además el número de funciones de base es 9 , de las cuales 8 definirán la matriz de ensamblaje final.

\section{Calculamos la matriz de Rígidez}

$$
A_{i j}^{k}=\int_{x_{k-1}}^{x_{k}}\left(p\left(\phi_{i}^{k}\right)^{\prime}\left(\phi_{j}^{k}\right)^{\prime}+r\left(\phi_{i}^{k}\right)\left(\phi_{j}^{k}\right)\right) d x
$$

Hallamos la matriz de rígidez para un subintervalo cualquiera :

$$
\begin{gathered}
A_{11}^{e}=\int_{x_{k-1}}^{x_{k}}\left[\left(\frac{1}{h}\right)^{2}+\left(\frac{x_{k-1}-x}{h}\right)^{2}\right] d x=\frac{1}{h}+\frac{h}{3} \\
A_{12}^{e}=A_{21}^{e}=\int_{x_{k-1}}^{x_{k}}\left[-\left(\frac{1}{h}\right)^{2}+\left(\frac{x_{k-1}-x}{h}\right)\left(\frac{x_{k}-x}{h}\right)\right] d x=-\frac{1}{h}+\frac{h}{6} \\
A_{22}^{e}=\int_{x_{k-1}}^{x_{k}}\left[\left(\frac{1}{h}\right)^{2}+\left(\frac{x-x_{k}}{h}\right)^{2}\right] d x=\frac{1}{h}+\frac{h}{3}
\end{gathered}
$$

Con esto tendremos que la matriz de rígidez para este elemento:

$$
\Omega_{k}: \quad A^{k}=\left(\begin{array}{cc}
\frac{1}{h}+\frac{h}{3} & -\frac{1}{h}+\frac{h}{6} \\
\frac{-1}{h}+\frac{h}{6} & \frac{1}{h}+\frac{h}{3}
\end{array}\right)
$$

Con $h=0,125$, específicamente para cada elemento tenemos:

$$
A^{1}=A^{2}=\cdots=A^{8}=\left(\begin{array}{cc}
8,041667 & -7,979167 \\
-7,979167 & 8,041667
\end{array}\right)
$$

Con las matrices halladas formamos la matriz global

$$
A=A^{1}+A^{2}+A^{3}+A^{4}+A^{5}+A^{6}+A^{7}+A^{8}
$$




$$
A=\left(\begin{array}{ccccccccc}
8,0416 & -7,9792 & 0 & 0 & 0 & 0 & 0 & 0 & 0 \\
-7,9792 & 16,0833 & -7,9792 & 0 & 0 & 0 & 0 & 0 & 0 \\
0 & -7,9792 & 16,0833 & -7,9792 & 0 & 0 & 0 & 0 & 0 \\
0 & 0 & -7,9792 & 16,0833 & -7,9792 & 0 & 0 & 0 & 0 \\
0 & 0 & 0 & -7,9792 & 16,0833 & -7,9792 & 0 & 0 & 0 \\
0 & 0 & 0 & 0 & -7,9792 & 16,0833 & -7,9792 & 0 & 0 \\
0 & 0 & 0 & 0 & 0 & -7,9792 & 16,0833 & -7,9792 & 0 \\
0 & 0 & 0 & 0 & 0 & 0 & -7,9792 & 16,0833 & -7,9792 \\
0 & 0 & 0 & 0 & 0 & 0 & 0 & -7,9792 & 8,0416
\end{array}\right)
$$

Calculamos el Vector de Carga

Donde el vector de carga esta formado por dos elementos

$$
\begin{gathered}
f_{1}^{k}=\int_{x_{k-1}}^{x_{k}} f \phi_{1}^{k} d x=\int_{x_{k-1}}^{x_{k}} x \operatorname{sen}(x)\left(\frac{x_{k}-x}{h}\right) d x \\
f_{2}^{k}=\int_{x_{k-1}}^{x_{k}} f \phi_{1}^{k} d x=\int_{x_{k-1}}^{x_{k}} x \operatorname{sen}(x)\left(\frac{x-x_{k-1}}{h}\right) d x
\end{gathered}
$$

Vector de Carga o fuerza

$$
F^{k}=\left[\begin{array}{l}
f_{1}^{k} \\
f_{2}^{k}
\end{array}\right]
$$

en el problema:

$$
\begin{array}{r}
f_{1}^{k=} \int_{x_{k-1}}^{x_{k}} x \operatorname{sen}(x)\left(\frac{x_{k}-x}{h}\right) d x=-\frac{x_{k} x_{k-1} \cos \left(x_{k-1}\right)}{h}+\frac{x_{k} \operatorname{sen}\left(x_{k}\right)}{h}+\frac{2 \cos \left(x_{k}\right)}{h}+\frac{x_{k} \operatorname{sen}\left(x_{k-1}\right)}{h} \\
+\frac{x_{k-1}^{2} \cos \left(x_{k-1}\right)}{h}-\frac{2 \cos \left(x_{k-1}\right)}{h}-\frac{2 x_{k-1} \operatorname{sen}\left(x_{k-1}\right)}{h} \\
f_{2}^{k=} \int_{x_{k-1}}^{x_{k}} x \operatorname{sen}(x)\left(\frac{x-x_{k-1}}{h}\right) d x=- \\
+\frac{x_{k} \operatorname{sen}\left(x_{k-1}\right)}{h}+\frac{x_{k} x_{k-1} \cos \left(x_{k}\right)}{h}-\frac{x_{k}^{2} \cos \left(x_{k}\right)}{h}+\frac{2 \cos \left(x_{k}\right)}{h} \\
h
\end{array}
$$

Con $h=0,125$, específicamente para cada vector de carga local tenemos:

\section{Vector $\mathbf{F}^{1}$}

para $x_{1}=0 ; x_{2}=0,125$

$$
F^{1}=\left(\begin{array}{l}
0,0001626 \\
0,0004874
\end{array}\right)
$$

\section{Vector $\mathbf{F}^{2}$}

para $x_{2}=0,125 ; x_{3}=0,250$

$$
F^{2}=\left(\begin{array}{c}
0,00117807 \\
0,002745
\end{array}\right)
$$

\section{Vector $\mathbf{F}^{3}$}

para $x_{3}=0,250 ; x_{4}=0,375$

$$
F^{3}=\left(\begin{array}{c}
0,052912 \\
0,0068650
\end{array}\right)
$$




\section{Vector $\mathbf{F}^{4}$}

para $x_{4}=0,375 ; x_{5}=0,500$

$$
F^{4}=\left(\begin{array}{c}
0,0105842 \\
0,0127178
\end{array}\right)
$$

\section{Vector $\mathbf{F}^{5}$}

para $x_{5}=0,500 ; x_{6}=0,625$

$$
F^{5}=\left(\begin{array}{l}
0,0174926 \\
0,0201184
\end{array}\right)
$$

\section{Vector $\mathbf{F}^{6}$}

para $x_{6}=0,625 ; x_{7}=0,750$

$$
F^{6}=\left(\begin{array}{c}
0,0257964 \\
0,0288302
\end{array}\right)
$$

\section{Vector $\mathbf{F}^{7}$}

para $x_{7}=0,750 ; x_{8}=0,875$

$$
F^{7}=\left(\begin{array}{l}
0,03522810 \\
0,03857103
\end{array}\right)
$$

\section{Vector $\mathbf{F}^{8}$}

para $x_{8}=0,875 ; x_{9}=1,000$

$$
F^{8}=\left(\begin{array}{l}
0,045478222 \\
0,049019205
\end{array}\right)
$$

Entonces el vector de carga será;

$$
\begin{gathered}
F=F^{1}+F^{2}+F^{3}+F^{4}+F^{5}+F^{6}+F^{7}+F^{8} \\
F=\left(\begin{array}{c}
0,0001626-p(0) y^{\prime}(0) \\
0,00226810 \\
0,00803630 \\
0,01744920 \\
0,03021040 \\
0,04591480 \\
0,06405830 \\
0,08404925 \\
0,04901920+p(1) y^{\prime}(1)
\end{array}\right)
\end{gathered}
$$

Debido a las condiciones de frontera $y(0)=2, y^{\prime}(1)=3$, se procede:

i) $y(0)=2$ : se anula la primera fila y columna de la matriz de ensamblaje, y el primer elemento del vector de carga. También en el vector de carga que queda, su primer elemento sufre una compensación.

ii) $y^{\prime}(1)=3$ : se cambia $A_{22}^{8}$ por $A_{22}^{8}+(1)(3)$ y se cambia $f_{2}^{8}+(1) y^{\prime}(1)$ por $f_{2}^{8}+(1)(3)$. Así tendremos: 


$A=\left(\begin{array}{cccccccc}16,0833 & -7,9792 & 0 & 0 & 0 & 0 & 0 & 0 \\ -7,9792 & 16,0833 & -7,9792 & 0 & 0 & 0 & 0 & 0 \\ 0 & -7,9792 & 16,0833 & -7,9792 & 0 & 0 & 0 & 0 \\ 0 & 0 & -7,9792 & 16,0833 & -7,9792 & 0 & 0 & 0 \\ 0 & 0 & 0 & -7,9792 & 16,0833 & -7,9792 & 0 & 0 \\ 0 & 0 & 0 & 0 & -7,9792 & 16,0833 & -7,9792 & 0 \\ 0 & 0 & 0 & 0 & 0 & -7,9792 & 16,0833 & -7,9792 \\ 0 & 0 & 0 & 0 & 0 & 0 & -7,9792 & 8,0416+3\end{array}\right)$

$$
A\left(\begin{array}{l}
w_{2} \\
w_{3} \\
w_{4} \\
w_{5} \\
w_{6} \\
w_{7} \\
w_{8} \\
w_{9}
\end{array}\right)=\left(\begin{array}{c}
0,00226810-A_{21}^{1}(2) \\
0,00803630 \\
0,01744920 \\
0,03021040 \\
0,04591480 \\
0,06405830 \\
0,08404925 \\
0,04901920+3
\end{array}\right)
$$

Como tenemos que $A_{21}^{1}=-7,9791667$, entonces:

$$
\left(\begin{array}{l}
w_{2} \\
w_{3} \\
w_{4} \\
w_{5} \\
w_{6} \\
w_{7} \\
w_{8} \\
w_{9}
\end{array}\right)=\left(\begin{array}{l}
2,0939 \\
2,2203 \\
2,3805 \\
2,5758 \\
2,8077 \\
3,0777 \\
3,3880 \\
3,7408
\end{array}\right)
$$

Con lo cual:

$$
\left(\begin{array}{l}
w_{1} \\
w_{2} \\
w_{3} \\
w_{4} \\
w_{5} \\
w_{6} \\
w_{7} \\
w_{8} \\
w_{9}
\end{array}\right)=\left(\begin{array}{c}
2,0 \\
2,0939 \\
2,2203 \\
2,3805 \\
2,5758 \\
2,8077 \\
3,0777 \\
3,3880 \\
3,7408
\end{array}\right)
$$

entonces:

$$
y(x)=w_{1} \phi_{1}+w_{2} \phi_{2}+w_{3} \phi_{3}+w_{4} \phi_{4}+w_{5} \phi_{5}+w_{6} \phi_{6}+w_{7} \phi_{7}+w_{8} \phi_{8}+w_{9} \phi_{9}
$$


Tenemos a continuación la gráfica de esta función (solución aproximada):

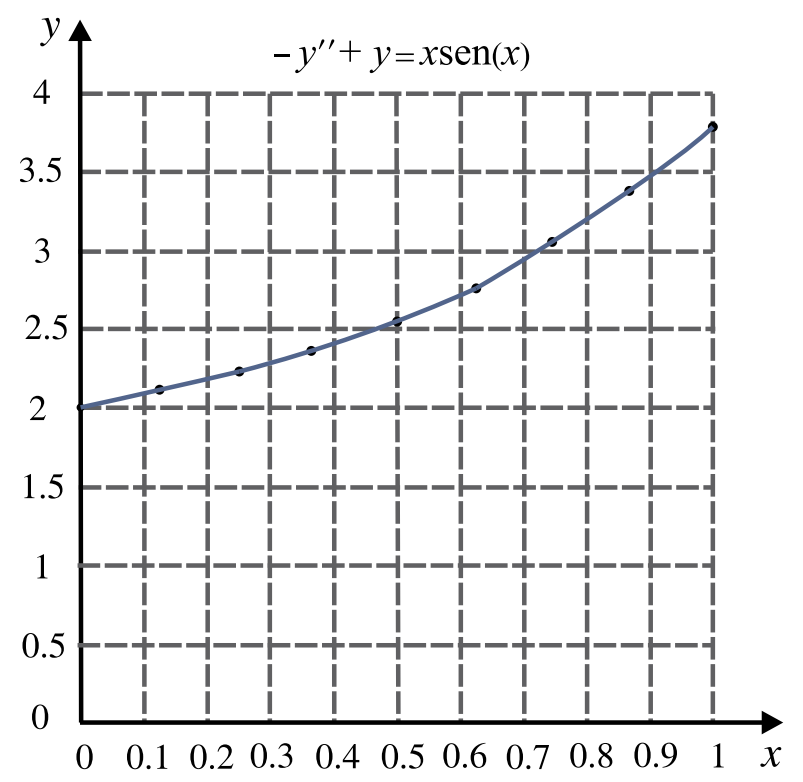

\section{Conclusiones}

1. Luego de haber visto todo el proceso podemos llegar a la conclusión de que toda EDO tiene solución pero esta no siempre la obtendremos de manera implícita, pero si podemos dar un bosquejo por medio de una solución aproximada la cual nos dará una idea de como se comportará nuestra solución real.

2. Mientras más funciones bases utilicemos nuestra solución se aproximará mejor a la solución exacta del problema que vamos a atacar.

3. Los elementos finitos lineales son una opción practica para poder aproximar de manera efectiva las soluciones al modelo planteado. 


\section{REFERENCIAS BIBLIOGRÁFICAS}

[1] Adams, R. A. (1975). Sobolev Spaces. Academic Press, New York.

[2] Atkison, K.; Han, W. (2000). Theoretical Numerical Analysis. Springer-Verlag.

[3] Brezis, H. (1984). Análisis Funcional, Teoría y Aplicaciones. Alianza Editorial, S.A. Madrid.

[4] Dunford, N.; Schwartz, J. T. (1958). Linear Operators. Interscience Publishers Inc., New York.

[5] Dautray, R.; Lions, J. (1988). Mathematical Analysis and Numerical Methode for Science and Technology. Springer - Verlag Berlin Heiderlberg.

[6] Jaime Muñoz. (2004). Teoria das Distribuições e Equações Diferenciais Parciais. Textos Avanzados-LNCC. Petrópolis-Rio de Janeiro.

[7] Luiz A. Medeiros. (1968). Tópicos de Análise Funcional. Instituto de Matemática, Univ. Federal de Pernambuco.

[8] Luiz A. Medeiros. (1989). A Integral de Lebesgue. Universidade Federal do Rio de Janeiro, Centro de Ciências Matemáticas e da Natureza, Instituto de Matemática.

[9] Schwartz, H. R. (1988). Finite Element Methods. Academic Press, New York.

[10] Walter R. (1973). Functional Analysis. McGraw-Hill, Inc.

[11] Yosida, K. (1980). Functional Analysis. 6th Ed., Springer-Verlag. 